\title{
Problemas a largo plazo de un programa hospitalario de cirugía sin ingreso
}

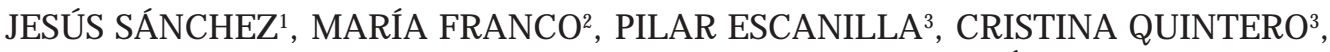 \\ SUSANA FRANCO ${ }^{3}$, ANA SANZ 3 , ALFREDO JIMÉNEZ4 \\ ${ }^{1}$ Servicio de Cirugía General y Digestiva, Hospital Comarcal de J aca. España. ${ }^{2}$ Servicio de Obstetricia y \\ Ginecología, Hospital Comarcal de Jaca. España. ${ }^{3}$ Servicio de Enfermería Quirúrgica, Hospital Comarcal de Jaca. \\ España. ${ }^{4}$ Coordinador de la U CMA del Hospital Clínico Universitario de Zaragoza. España.
}

\begin{abstract}
Resumen
E n la presente revisión, y apoyándonos en nuestra experiencia en el Hospital Comarcal de Jaca, intentaremos poner de relieve y discutir los beneficios y los problemas que generan las unidades de cirugía sin ingreso. A nalizaremos los distintos tipos de programas ambulatorios y los modelos de unidad quirúrgica, mostrando sus ventajas e inconvenientes. Por último, intentaremos proponer diversas líneas para mejorar la calidad ofertada, la satisfacción del usuario y la relación coste / efectividad de los procesos quirúrgicos.
\end{abstract}

Palabras clave: Cirugía ambulatoria; técnicas quirúrgicas; calidad de la atención en salud; servicio de cirugía en hospital.

\begin{abstract}
Long term problems with an outpatient surgery hospital program Abstract

In this paper we will try to stand out and discuss the benefits and difficulties generated by outpatient units according to our experience. We will analyse the different types of surgical programs, showing advantages and drawbacks. Finally, we will propose several ways in order to improve quality assurance, user's satisfaction and cost / effectiveness relation of surgical processes.
\end{abstract}

Key words: Ambulatory surgery; surgery, operative; quality of health care; surgery department, hospital.

\section{INTRODUCCIÓN}

Cuando a principios de los años 90 se puso en marcha los programas pioneros de cirugía sin ingreso en nuestro país, nadie, ni tan siquiera los propios cirujanos implicados, podía adivinar la magnitud del impacto que esta nueva forma de trabajar tendría en nuestra actividad quirúrgica cotidiana $\left({ }^{1}\right)$.

Resulta difícil explicar cómo en apenas un decenio se ha pasado de aquella tímida cirugía de corta estancia (CCE) a practicar colecistectomías laparoscópicas ambulatorias.

U n fenómeno destacable de aquellos inicios, tal vez irrepetible, fue la voluntad única y decidida de impulsar la cirugía mayor ambulatoria (CM A) en nuestro Sistema $\mathrm{N}$ acional de Salud; voluntad manifestada al unísono por los responsables políticos, gestores hospitalarios y facultativos.

Pero lo más sorprendente, si cabe, de todo el proceso de implantación de Ia CMA ha sido el cambio de mentalidad que han experimentado los enfermos. Si en los inicios era el cirujano el que debía persuadir a su paciente de las ventajas de la CMA, ahora son ellos los que demandan (cuando no exigen) ser operados ambulatoriamente.

A ctualmente, Ia CM A vive su plena madurez, mientras que los profesional es que la adoptamos de manera entusiasta atravesamos un periodo de reflexión, evaluación y, por qué no decirlo, de desánimo, en no pocas ocasiones. 
En las "V J ornadas A ragonesas sobre Cirugía A mbulatoria", que tuvieron lugar en la capital de nuestra Comunidad A utónoma, Zaragoza, durante la primavera del año 2003, pudimos reunirnos especialistas de todos los centros hospitalarios de A ragón con programas de CM A en marcha. Allí pudimos compartir y constatar los problemas aparejados al mantenimiento de una actividad ambulatoria. L as dificultades de todo tipo se incrementaban de manera exponencial en aquellos centros que no disponen de unidades integradas con buen diseño 0 unidades separadas, con hábitat, personal, material y organización específicos ( ${ }^{2}$ ).

Para poder determinar las carencias de un programa de CMA debemos establecer, apriorísticamente, los requisitos que lo caracterizan. La unidad de cirugía sin ingreso (UCSI) o de cirugía mayor ambulatoria (UCM A ), ya que ambos términos son sinónimos, se define como una organización de profesionales sanitarios que ofrece una asistencia multidisciplinar a procesos quirúrgicos susceptibles de ser ambulatorios, con tres condicionantes $(1,3)$ :

Pautas de funcionamiento estandarizadas mediante protocolos de consenso, aceptados por todos los estamentos.

- Unas instalaciones específicas e independientes del circuito habitual de los enfermos quirúrgicos ingresados. Estas estructuras deben estar dotadas de medios materiales que garanticen la labor del profesional y la comodidad del usuario.

- Una organización autónoma y perfectamente establecida: liderazgo, equipo, supervisión, política de incentivos al personal, control de calidad mediante auditorías externas y encuestas al usuario, etc. De esta manera, se garantiza el funcionamiento, la eficiencia y la eficacia de los recursos invertidos.

Como paso previo a la obtención de estos logros ideales, algunos autores han propuesto una línea de continuidad que se iniciaría con la creación de un programa preliminar de C CE $\left({ }^{4}\right)$. Posteriormente, se produciría un progreso hacia una unidad ambulatoria integrada, a la que se dotaría de un circuito propio de pacientes. EI objetivo último debe ser el diseño de un programa sin ingreso con personal, circuito de pacientes y ámbito arquitectónico propios. E sta política de "implantación lenta" de Ias UCSI, permite una vía opcional y un periodo de adaptación para profesional es y usuarios cuando el volumen de pacientes o las disponibilidades económicas son escasas. Pero no debemos engañarnos: detener esta progresión lógica en sus fases iniciales, tras recoger los primeros réditos, puede suponer un éxito temporal, pero, sin duda, un fracaso a largo plazo por agotamiento del modelo.

En el presente artículo, y apoyándonos en nuestra propia experiencia en el Hospital Comarcal de Jaca, intentaremos poner de relieve y discutir los problemas asociados a las U CSI integradas, que nacieron poco menos que como un experimento y hoy en día se debaten entre una demanda social desbordada, unos medios humanos y materiales precarios y una cierta desatención por parte de los gestores que han puesto sus miras y recursos en actividades más novedosas y mediáticas.

\section{DISEÑO DE UNA UCSI: IM PLICACIONES A LARGO PLAZO}

Desde principios de los años 90 (primera UCMA en funcionamiento en España en el Hospital de Viladecans), en cada territorio del Estado y en cada hospital se planificó de diversa manera la puesta en marcha de los programas de cirugía sin ingreso, intentando dar respuesta a múltiples realidades demográficas, económicas, arquitectónicas, entre otras.

Diseñar adecuadamente una U CSI requiere un detallado estudio preliminar que incluye un análisis demográfico de la población a la que atiende el centro y una valoración realista de la demanda teórica de CMA y de la cartera de servicios. Con todo ello, se estudiará las necesidades de personal y equipamiento, además de construir o adaptar los espacios arquitectónicos $\left({ }^{5}\right)$.

Otra decisión a tomar es definir el modelo de unidad que queremos implantar. Partiendo de la premisa que las unidades de CMA 
extrahospitalarias y las free-standing (independientes administrativa y arquitectónicamente) quedan al margen de este estudio, podremos elegir entre $(3,5)$ :

\section{Ambulatorización de procesos} seleccionados. Permite, con un mínimo desembolso, aprovechar espacios infrautilizados y complementar la actividad de un servicio quirúrgico. Ofrece resultados inmediatos, pero resulta inviable como modelo sostenido. Puede asumirse como un escalón superior a un programa de CCE, pero no es una auténtica UCM A.

2. Unidad integrada. Los pacientes ambulatorios comparten el bloque quirúrgico con los hospitalizados, pero se crean vías clínicas propias, espacios adecuados (readaptación al medio) y se dota a la unidad de algún personal propio, por ejemplo en enfermería o administración ( $\left.{ }^{6}\right)$.

3. Unidad separada. Creada como un servicio independiente dentro del hospital, con sus quirófanos, estructuras arquitectónicas y administrativas.

Por último, el diseño se completa con el establecimiento de los criterios de inclusión/ exclusión de los pacientes susceptibles de ser tratados ambulatoriamente y la selección de los procedimientos por especialidad que van a ser tratados, esto es, la cartera de servicios $\left({ }^{7}\right)$.

No cabe duda que todas esas decisiones iniciales marcarán el devenir de la futura U CSI y serán determinantes a la hora de condicionar el éxito o el fracaso a largo plazo.

\section{EXPERIENCIA Y RESULTADOS DE LA UCSI DEL HOSPITAL DE JACA}

El Hospital Comarcal de Jaca es un centro sanitario cuya titularidad pertenece a la A dministración Pública (Diputación General de A ragón y Ayuntamiento de Jaca) y que se constituye, desde el año 1995, como un Consorcio Hospitalario financiado a cargo de Ios Presupuestos Generales de la Comunidad A utónoma, pero con un modelo privado de gestión. El centro proporciona cobertura sanitaria a una población de unos 35000 habitantes, caracterizada por el envejecimiento de la pirámide poblacional, la dispersión en pequeños núcleos rurales alejados y mal comunicados y la estacionalidad, al tratarse de una zona turística (cuatro grandes centros invernales en su ámbito de influencia).

A ntes de 1995, las intervenciones quirúrgicas eran facturadas en base a los días de estancia postoperatoria. Con el nuevo Contrato Programa, se instauró la facturación mediante un montante fijo por proceso; por este motivo, el objetivo prioritario de la Dirección del Hospital fue el acortamiento de la estancia postoperatoria y, secundariamente, hacer uso de un quirófano y varias dependencias adyacentes infrautilizadas. Respondiendo a estas demandas, los distintos servicios quirúrgicos pusimos en marcha un programa preliminar de CCE para, en una segunda fase, intentar el desarrollo de una auténtica UCSI integrada en el bloque quirúrgico $\left.{ }^{8}\right)$. Como efectos beneficiosos del programa de cirugía sin ingreso reseñaremos $\left({ }^{9}\right)$ :

- El objetivo de reducir las estancias hospitalarias de los procesos concertados se ha cumplido ampliamente. A demás, el efecto de la CM A ha contagiado a la cirugía urgente y programada no ambulatorizable, por su complejidad. En la Figura 1 puede observarse el continuo descenso de la estancia media de los servicios quirúrgicos del hospital.

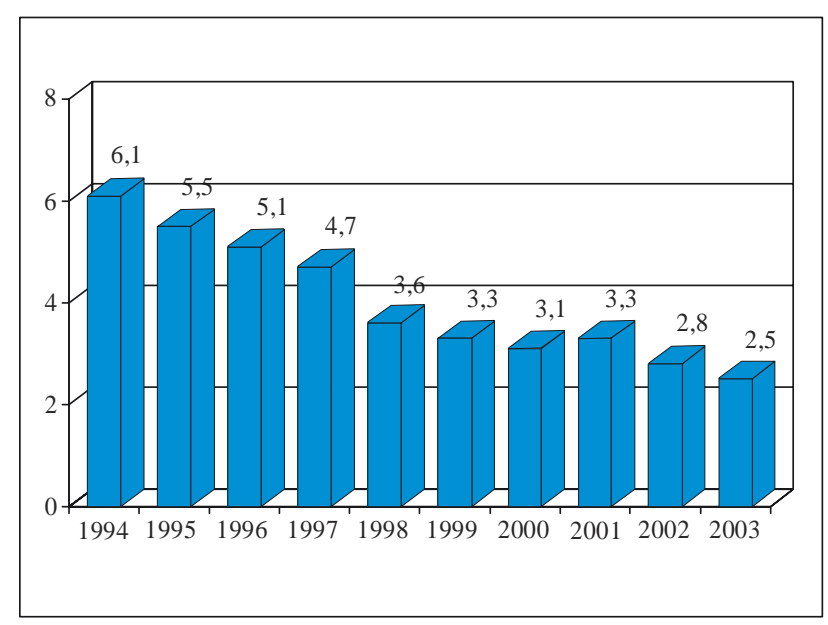

Figura 1. Estancia media de los servicios quirúrgicos del Hospital Comarcal de Jaca. 
- La CMA ha aumentado su importancia de forma paulatina, y supone actualmente un $50 \%$ de la cirugía mayor programada, en paridad con la cirugía susceptible de ingreso (Tabla 1 y Figura 2). N uestro cociente cirugía ambulatoria / no ambulatoria puede ser considerada dentro de los límites óptimos propuestos por los organismos sanitarios nacionales e internacionales, que lo cifran entre un 50 y $60 \%\left({ }^{3}\right)$.

- La magnitud de las intervenciones incluidas en el programa ha ido en progresión y, actualmente, várices, hernias, artroscopias, etc., sustituyen a otros procedimientos que ahora se las contabiliza como cirugía menor ambulatoria (Tabla 2). Este tipo de cirugía menor de una cierta entidad (vasectomía, cirugía plástica en cáncer de piel, gangliones, uña encarnada, etc.), ha experimentado un crecimiento importante $y$, dado que requiere personal, medios y quirófano adecuados, representa un problema añadido para la planificación de la U CSI $\left({ }^{10}\right)$.

- L a falta de anestesistas, endémica en nuestro Sistema Sanitario, ocasiona trastornos constantes de la actividad, ya que deben atender el área obstétrica, la cirugía programada y las guardias localizadas.

- El éxito y la el evadísima demanda del programa de cirugía de la catarata ha ocasionado una escasez crónica de las sesiones quirúrgicas disponibles para el resto de los servicios.

- El desarrollo de una vía clínica de la catarata demuestra la gran capacidad organizativa del personal de Ia UCSI y ha redundado en la calidad ofertada y en un mayor ahorro económico.

- La satisfacción expresada por nuestros usuarios es excel ente, según se desprende de una encuesta externa de calidad.

Pese a todos los logros alcanzados durante estos años, hemos comprobado la persistencia de diversos problemas. En nuestra opinión, la viabilidad a largo plazo del programa de CM A depende de la adecuada corrección de estos defectos por las A utoridades Sanitarias:

- Los enfermos de Ia CMA siguen compartiendo los quirófanos y la readaptación postanestésica con la cirugía programada con ingreso.

- A pesar de las demandas, no hemos conseguido la construcción de una sala de readaptación al medio independiente, dotada de las necesarias comodidades para el enfermo y sus acompañantes.

- No hemos conseguido la contratación de personal específico para la UCSI, en especial de tipo administrativo.

- Todavía no disponemos de una documentación común, avalada por el sello corporativo del Hospital de Jaca: consentimientos informados, folletos informativos para los usuarios, hojas de instrucciones posquirúrgicas, etc. Cada servicio se ve en la necesidad de elaborar sus propios documentos.

Tabla 1. Actividad de la UCSI del Hospital Comarcal de Jaca 1998 - 2003.

\begin{tabular}{rrcrrrrr}
\hline & Cirugía & Traumatología & Ginecología & Oftalmología & CMA & CCI & $\%$ CMA \\
\hline 1998 & $72(38 \%)$ & $55(29 \%)$ & $13(7 \%)$ & $50(26 \%)$ & 190 & 234 & $45 \%$ \\
1999 & $65(34 \%)$ & $17(9 \%)$ & $12(6 \%)$ & $99(51 \%)$ & 193 & 196 & $50 \%$ \\
2000 & $58(28 \%)$ & $23(14 \%)$ & $9(4 \%)$ & $121(54 \%)$ & 211 & 230 & $48 \%$ \\
2001 & $55(19 \%)$ & $22(8 \%)$ & $15(5 \%)$ & $198(68 \%)$ & 290 & 288 & $50 \%$ \\
2002 & $67(24 \%)$ & $36(13 \%)$ & $11(4 \%)$ & $166(59 \%)$ & 280 & 314 & $47 \%$ \\
2003 & $89(25 \%)$ & $29(8 \%)$ & $10(3 \%)$ & $225(64 \%)$ & 353 & 309 & $53 \%$ \\
Total & $406(27 \%)$ & $182(12 \%)$ & $70(4 \%)$ & $859(57 \%)$ & 1517 & 1571 & $49 \%$ \\
\hline
\end{tabular}

UCSI: Unidad de cirugía sin ingreso; CMA: Cirugía mayor ambulatoria; CCI: Cirugía mayor programada con ingreso. Se observa el número de casos ambulatorizados, los porcentajes por servicio y de la CMA con respecto al CCI. 


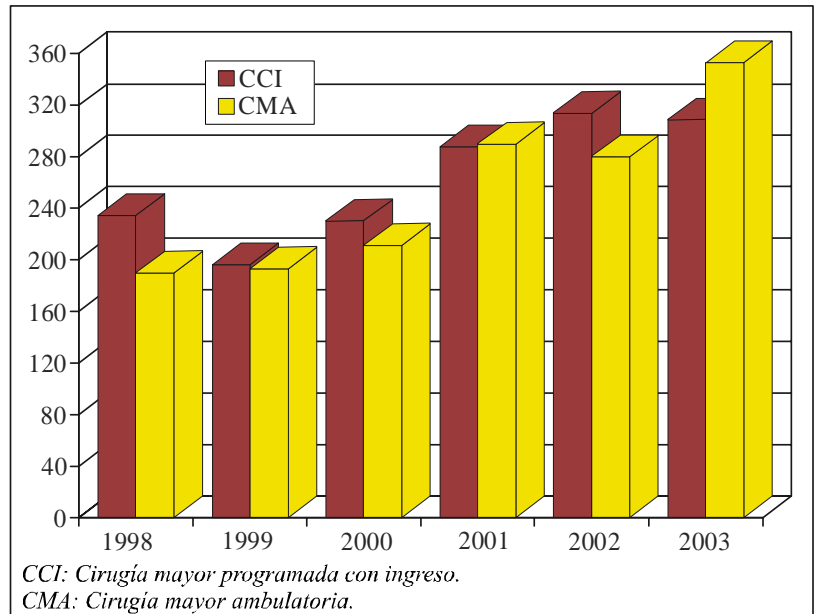

Figura 2. Cirugía mayor programada, en paridad con la cirugía susceptible de ingreso.

- No se ha nombrado una persona responsable del programa que coordine las actuaciones de los servicios e imponga criterios de calidad $y$ de orden.

- A ctualmente ningún facultativo, enfermera, auxiliar o administrativo que participa en el programa de CMA recibe incentivo alguno (económico, días festivos, facilidades para asistencia a cursos, etc.) aunque lleve a cabo actividades fuera de su horario o que excedan sus funciones.

Consideramos que el futuro de la CMA en nuestro centro, tras el lógico periodo de puesta en marcha y adaptación del personal y los usuarios que consideramos total mente superado, se encuentra en un momento decisivo. Las necesarias inversiones deben encaminarse a financiar los siguientes aspectos:

- Creación de una auténtica UCSI. Para ello se precisa la completa remodelación del área de urgencias y el bloque quirúrgico, con la puesta en marcha de un quirófano propio para CMA y un circuito independiente de los pacientes ambulatorios.

- Conseguir que los servicios implicados actúen de forma coordinada en cuanto a documentación, objetivos, planificación de las sesiones quirúrgicas, revisión crítica de los resultados, etc. Para ello, es necesaria la creación del puesto de coordinador del programa de CMA.

Tabla 2. Procesos quirúrgicos de CMA más frecuentes distribuidos por especialidades en el periodo 2000 - 2003.

\begin{tabular}{|c|c|c|c|c|c|c|}
\hline Servicio & Procedimiento & 2000 & 2001 & 2002 & 2003 & Total \\
\hline \multirow[t]{5}{*}{ Cirugía } & Varices & 17 & 22 & 27 & 36 & 102 \\
\hline & Hernia & 14 & 11 & 16 & 15 & 56 \\
\hline & Fimosis & 9 & 9 & 8 & 11 & 37 \\
\hline & Proctología & 7 & 5 & 8 & 13 & 33 \\
\hline & Injertos / Colgajos & 5 & 4 & 3 & 5 & 17 \\
\hline Cirugía / Ginecológica & Exéresis tumor benigno mama & 3 & 3 & 4 & 4 & 14 \\
\hline \multirow[t]{4}{*}{ Ginecología } & Biopsia de mama & 4 & 6 & 5 & 7 & 22 \\
\hline & Legrado / Histeroscopia & 2 & 3 & 2 & 2 & 9 \\
\hline & Conización cervical & 2 & 3 & 1 & - & 6 \\
\hline & Quiste de Bartholino & 1 & 1 & 2 & 1 & 5 \\
\hline \multirow[t]{5}{*}{ Traumatología } & Retirada material osteosíntesis & 7 & 11 & 8 & 3 & 29 \\
\hline & Ganglión en muñeca & 8 & 6 & 6 & 7 & 27 \\
\hline & Artroscopia & - & - & 12 & 14 & 26 \\
\hline & Túnel del carpo & 3 & 1 & 5 & 3 & 12 \\
\hline & Dupuytren & 1 & - & 2 & - & 3 \\
\hline Todos servicios & Otros procedimientos & 7 & 6 & 5 & 7 & 25 \\
\hline
\end{tabular}

CMA: Cirugía mayor ambulatoria. 
- Dotación de personal facultativo (especialistas, enfermeras y auxiliares) integrados voluntariamente en el proyecto y debidamente recompensados por su plena dedicación al mismo.

- Garantizar la formación continuada del personal, facilitando la asistencia a congresos y cursos de formación o actualización.

- Personal administrativo informado del funcionamiento de Ia UCSI, e implicado positivamente en la actividad de la misma.

Como conclusión, estamos convencidos que la puesta en marcha de una UCSI es una sólida apuesta de futuro para cualquier centro hospitalario ( $\left.{ }^{11}\right)$. Los beneficios de la CM A son capaces de impregnar toda la actividad quirúrgica, disminuyendo las estancias hospitalarias y permitiendo agilizar las listas de espera, verdadero azote de los sistemas sanitarios de titularidad pública. Los efectos beneficiosos pueden ser más intensos, si cabe, en los hospitales comarcales de mediano o pequeño tamaño, en los que un elevado porcentaje de su actividad operatoria es susceptible de ser ambulatorizada.

Pese a todo, debemos recordar que la CM A no es una forma de recortar gastos económicos, sino más bien una nueva mentalidad de trabajo encaminada a lograr la comodidad y el bienestar del paciente y, de forma secundaria, mejorar la relación coste/efectividad en los procesos quirúrgicos ( $\left.{ }^{12}\right)$.

$V$ alorando nuestra experiencia, tras un periodo razonable de puesta en marcha, corrección de desajustes y optimización de los recursos materiales y humanos los buenos resultados de una U CSI están garantizados, todo ello con una inversión inicial mínima $\left({ }^{13}\right)$. Los profesionales implicados no debemos permitir que estos logros excepcionales a corto plazo impidan el desarrollo ulterior hacia modelos más evolucionados D ebemos reclamar, como mínimo, la creación y dotación de unidades integradas, pero cuyo ámbito de trabajo y estructura organizativa mantengan su independencia del resto de la actividad quirúrgica programada del centro.

Por último, consideramos indispensable que la fidelidad y entrega de las personas implicadas en la CM A sea recompensada por los gestores, favoreciendo su formación continuada y la adecuada promoción profesional.

\section{REFERENCIAS BIBLIOGRÁFÍCAS}

1. Vila Blanco JM. Desarrollo e implantación de la CM A en E spaña: evolución en los hospitales del I N SA LUD. Cir Esp. 2004;76:177-83.

2. Jiménez A, Resa J, Cerdán R, Lagos J, Escartín A, González M. Dificultades para la implantación de la Cirugía M ayor A mbulatoria en un hospital público. Cir Esp. 1995;58:476-9.

3. Porrero JL (ed). Cirugía M ayor A mbulatoria. M anual Práctico. M adrid: Ediciones D oyma S. A .; 1999.

4. Villar del M oral JM, García M artos JB, M artín Ruiz de la Herran J M, García Rubio J, Hernández García J M, Ruiz Lorenzo PM, Torres A Icalá JT. ¿Qué aporta la cirugía de corta estancia a las unidades de Cirugía M ayor A mbulatoria?. Cir M ay A mb. 1998;3:272-6.

5. Sánchez Cabezudo, Díaz Guerra C, Porrero Carro JL. Diseño y estructuras de las unidades de Cirugía Mayor A mbulatoria. Cir M ay A mb. 1998; 3:195-8.

6. Diego M, A ndreu J, García P, Serrano P, Ferrer R, M oltó $M$, et al. Cirugía A mbulatoria en una unidad integrada en un Hospital. Desarrollo y evaluación de la misma. Cir Esp. 1996; 59:236-40.

7. M arsal F, Giner M. Evolución de los criterios de selección en un programa de Cirugía M ayor A mbulatoria. Cir Esp. 1995; 57:452-5.

8. Sánchez B eorlegui J, García Calleja J L, L amata H ernández F, Fernández Ruiz M , Córdoba Díaz De L aspra E, Ceballos A lonso C. Puesta en marcha y resultados preliminares de la Unidad de Cirugía sin Ingreso del Hospital Comarcal de Jaca. Cir M ay A mb. 1999;4:324-8.

9. Sánchez B eorlegui J, García Calleja JL, C erezo García M M , Burgos Hernández J M , L amata Hernández F, Saavedra D el Rosario E. Evaluación del impacto de la Cirugía A mbulatoria en la actividad quirúrgica de un hospital comarcal. Cir M ay A mb. 2002; 7:78-80.

10. Ibáñez F, L ucero JA, Naranjo B, Díaz C, Pazos M, Font M. Cirugía ambulatoria menor. N uestra experiencia en 2.600 pacientes. Cir May A mb. 1998;3:56-7.

11. Raich Brufrau M . Cirugía M ayor A mbulatoria. Un beneficio para todos. Cir M ay A mb. 2001;6:9-10.

12. Jiménez $A$, Pemán $M J$, González R, A ripes $P$, Algora $C$, Gracia M. Encuesta de satisfacción en Cirugía A mbulatoria: instrumento para detectar puntos débiles y monitorizar mejoras. Cir M ay A mb. 2002; 7:164-72.

13. Vila Blanco J M. Gestión de costes mediante la implantación de una unidad de Cirugía Mayor A mbulatoria. Cir M ay A mb. 2002; 7:66-72.

Correspondencia:

Dr. J esús Sánchez Beorlegui

Calle Pedregales $\mathrm{n} 0$ 9, 50 $\mathrm{E}$.

26006 Logroño. España

Correo-e: js_beorlegui @hotmail.com 